\title{
Gastrointestinal Stoma Stenosis
}

National Cancer Institute

\section{Source}

National Cancer Institute. Gastrointestinal Stoma Stenosis. NCI Thesaurus. Code C78628.

Narrowing of the lumen of a gastrointestinal stoma. 